\title{
Uneven distribution of potential triplex sequences in the human genome In silico study using the R/Bioconductor package triplex
}

\author{
Matej Lexa ${ }^{1}$, Tomáš Martínek ${ }^{2}$ and Marie Brázdováa ${ }^{3}$ \\ ${ }^{1}$ Faculty of Informatics, Masaryk University, Botanická 68a, 60200 Brno, Czech Republic \\ ${ }^{2}$ Faculty of Information Technology, Brno Technical University, Božetěchova 1/2, 61266 Brno, Czech Republic \\ ${ }^{3}$ Biophysical Institute of the Czech Academy of Sciences, Královopolská 135, 61265 Brno, Czech Republic \\ lexa@fi.muni.cz,martinto@fit.vutbr.cz,maruska@ibp.cz.
}

Keywords: Human genome, DNA sequence, Non-B-DNA, Triplex, Bioconductor, Repetitive sequences, Mobile DNA, Lexicographically minimal rotation

Abstract: $\quad$ Eukaryotic genomes are rich in sequences capable of forming non-B DNA structures. These structures are expected to play important roles in natural regulatory processes at levels above those of individual genes, such as whole genome dynamics or chromatin organization, as well as in processes leading to the loss of these functions, such as cancer development. Recently, a number of authors have mapped the occurrence of potential quadruplex sequences in the human genome and found them to be associated with promoters. In this paper, we set out to map the distribution and characteristics of potential triplex-forming sequences (PTS) in the human genome sequence. Using the R/Bioconductor package triplex, we found these sequences to be excluded from exons, while present mostly in a small number of repetitive sequence classes, especially short sequence tandem repeats (microsatellites), Alu and combined elements, such as SVA. We also introduce a novel way of classifying potential triplex sequences, using a lexicographically minimal rotation of the most frequent k-mer to assign class membership automatically. Members of such classes typically have different propensities to form parallel and antiparallel intramolecular triplexes (H-DNA). We observed an interesting pattern, where the predicted third strands of antiparallel H-DNA were much less likely to contain a deletion than their duplex structural counterpart than were their parallel versions.

\section{INTRODUCTION}

Eukaryotic genomes are rich in sequences capable of forming non-B DNA structures. Cruciform, slipped, triplex or quadruplex DNA has been recognized as a factor in several important biological processes or functions (Buske et al., 2011) (Bacolla and Wells, 2004). Non-B DNA is often found close to recombination hotspots and is thought to aid genomic instability and evolution (Zhao et al., 2010). The structures it forms have the ability to modulate replication (Dixon et al., 2008), transcription (Rich and Zhang, 2008) or translation (Arora et al., 2008) of DNA/RNA by mechanisms that may have their origins in times when nucleic acids dominated all life processes. These structures are expected to play important roles in natural regulatory processes at levels above those of individual genes, such as whole genome dynamics or chromatin organization (Sarkies et al., 2012) (Maizels and Gray, 2013), as well as in processes leading to the loss of these functions, such as cancer development.
For example, a recent study has shown that an interplay between G4-quadruplexes, FANCJ protein and DNA replication in cells influences the formation of euchromatin versus heterochromatin after the replication stage (Schwab et al., 2013). Ability to form $\mathrm{H}-\mathrm{DNA}$ is often associated with recombinational and mutational hotspots in the human genome (Akman et al., 1991).

Recently, a number of authors have mapped the occurrence of potential quadruplex sequences in the human genome and other eukaryotic genomes. They found them to be associated with promoters and certain classes of repeat elements (Savage et al., 2013) (Lexa et al., 2013). Possibilities of searching for nonB DNA (Cer et al., 2011) and specifically triplex/HDNA exist as well (Buske et al., 2012) (Hon et al., 2013). In this paper, we map the distribution and characteristics of potential triplex-forming sequences (PTS) in human genomic DNA as detected/predicted using the $\mathrm{R} /$ Bioconductor package triplex. 


\section{SOFTWARE AND METHODS}

To analyze the human genome, or other sequence sets, we employed the R/Bioconductor framework, which has now matured to the point, where we can use $\mathrm{R}$ to represent biological sequences, search these sequences, represent the search results, analyze them statistically and visualize the results of the searches and the statistical analysis. All this can be done with relatively straightforward scripts, using a handful of well integrated R/Bioconductor software packages (Lawrence et al., 2013).

\subsection{R/Bioconductor packages used in this study}

Biostrings String objects representing biological sequences, and matching algorithms (Pages et al., 2013)

BSgenome Infrastructure for Biostrings-based genome data packages (Pages, 2013)

BSgenome.Hsapiens.UCSC.hg19 Homo sapiens (Human) full genome (UCSC version hg19)

biomaRt Interface to BioMart databases (e.g. Ensembl, COSMIC ,Wormbase and Gramene) (Durinck et al., 2009)

triplex Search and visualize intramolecular triplexforming sequences in DNA (Hon et al., 2013)

GenomicRanges Representation and manipulation of genomic intervals (Aboyoun et al., 2013)

\subsection{General triplex detection pipeline}

All types of potential triplexes (parallel and antiparallel) were identified in the human genome using the Bioconductor triplex package (Hon et al., 2013). We used the unmasked sequence from BSGenome.Hsapiens.UCSC.hg19 package in all analyses. Only potential triplexes with $\mathrm{P}$ value less than or equal 0.05 were considered for further analysis. A GFF file with all the identified potential triplexes is available at http://fi.muni.cz/ rexa/triplex/hsapiens_pts.gff

\subsubsection{Analysis of coding and non-coding regions}

Information about genes was obtained from the Ensembl database using its Biomart interface. Only coding genes at chromosomes 1-22, $\mathrm{X}$ and $\mathrm{Y}$ were considered (roughly $20 \mathrm{k}$ genes) and only their coding transcripts were selected for analysis (roughly 80k transcripts). Data about exons of selected transcripts were downloaded from Ensembl database and used for identification of promoters, introns, coding regions (CDS), 5'UTR, 3'UTR and intergenic regions. All this information was stored as individual tracks (GRanges objects). For the purpose of this study, promoters were defined as $1000 \mathrm{bp}$ regions upstream of the coding sequence (flanking the 5 ' end). Intergenic regions were identified as a complement to coding transcripts supplemented with promoters. In the next step we found the overlaps between triplexes and all prepared tracks. If a given triplex fell into more than one type of region (e.g. triplex is part of CDS and intron simultaneously) the triplex was counted in each overlapping region. Finally, we compared the results with numbers expected if positioning of potential triplexes was random. The expected values can be calculated from the percentage of genome covered by a certain type of region (see equations 1-5 below).

\subsubsection{Analysis of regions composed of repeats}

Information about different types of repeating sequences was obtained from the UCSC Table Browser, specifically from the Repeat Masker track (Karolchik et al., 2004). Data records were organized into 26 classes and 56 families covering both genes (coding and non-coding) and intergenic regions. At first, we analysed the number of potential triplexes in regions with and without repeats in genes and intergenic areas. As the majority of PTS were located in regions with repeats, we performed a detailed study of PTS overlapping individual repeat classes and families. In this experiment we focused on the number of repeats (in a given class or family) containing at least one triplex. The measured values were compared with numbers expected to be obtained at random.

We were also interested in potential triplexes occurring in close proximity to repeats. Therefore we extended all repeat regions with flanking areas (100bps at both ends) and repeated the analysis including these expanded areas.

\subsubsection{Calculation of expected values}

Expected values were calculated as the number of repeats (of given class/family) that would contain at least one triplex by random choice. This calculation consists of the following steps:

1. Number of triplexes $N_{\text {TrRep }}$ that would fall into a given class or family by random is calculated using equation 1 .

$$
N_{\text {TrRep }}=\frac{\sum_{\text {rep } \in \text { class }} \operatorname{len}(\text { rep })}{\operatorname{len}(\text { genome })}
$$


2. For each repeat $r e p_{s e l}$ of a given class/family:

(a) The probability that a randomily selected triplex is placed ouside of a given repeat is calculated using equation 2 .

$$
P_{\text {RepComp }}=\frac{\left(\sum_{\text {rep } \in \text { class }} \operatorname{len}(\text { rep })\right)-\operatorname{len}\left(\text { rep }_{\text {sel }}\right)}{\sum_{\text {rep } \in \text { class }} \operatorname{len}(\text { rep })}
$$

(b) Next, the probability that all triplexes of a given class/familly are placed outside of a given repeat is calculated using equation 3 .

$$
P_{\text {RepCompAll }}=\left(P_{\text {RepComp }}\right)^{N_{T r R e p}}
$$

(c) Finaly, the probility that at least one triplex falls into a given repeat is calculated using equation 4.

$$
P_{\text {Rep }}=1-P_{\text {RepCompAll }}
$$

3. The overall number of repeats that would contain at least one triplex by random choice is calculated as a sum of all probabilities calculated in the previous step (see equation 5).

$$
N_{\text {Rep }}=\sum_{\text {Rep } \in \text { class }} P_{\text {Rep }}
$$

Please note that in equations 1 and 2 we use a sum for expression of area occupied by repeats. In fact the real calculation is slightly more complex because the overlapping repeat regions have to be considered as well. Concretely, if two repeats of the same class or family overlap each other then the overlapping part is counted only once in that sum.

Expected values for repeats supplemented with flanking areas are calculated analogically.

\subsubsection{Analysis of non-coding genes}

Data about non-coding genes were obtained from the Ensembl database using the Biomart interface (roughly 40k genes). All genes were split into 26 categories based on their biotype (e.g. lincRNA, pseudogene, miRNA, scRNA, etc.) The same type of analysis, which was performed for repetitive sequences, was applied for non-coding genes as well.

All types of experiments were applied separately on a set of parallel, antiparallel and all types of triplexes (parallel and antiparallel together).

\subsection{Classification of potential triplex sequences}

H-DNA often forms in sequences that contain simple repetitions. The type of triplex that forms (e.g. parallel or antiparallel) often depends on the particular kind of repeat present. We therefore decided to classify the identified triplexes by the prevailing $k$-mers present in their sequences. Although different values of $k$ will serve the purpose of classification, we selected $k=6$ as a value which is not too high since high values of $k$ would produce a large number of categories that would be difficult to follow. Because $k=6$ is also the lowest number that can capture well both, periods of 2 and 3, we chose this value for all calculations in this study.

The prevailing $k$-mers for identical classes of sequences will sometimes differ, because in a periodic sequence, all rotations of the repeated sequence monomer will have similar chance of becoming the most prevalent $k$-mer. The precise result will depend on subtle changes in the sequence. For example, for $k=2$, the sequence $G C G C G C C G C G C$ will have 4 CG dinucleotides and $5 \mathrm{GC}$ dinucleotides, we would therefore label this sequence as "GC". A very similar sequence $C G C G C G G C G C G$ with one $C->G$ substitution and one nucleotide moved from the end to the front has 5 CGs and 4GCs and would therefore be labeled as "CG".

In situations were several rotations of a string may represent the same feature, we can deterministicaly choose one of the variants (rotations) to represent all of them. One way of choosing the representative string (hexamer) is to choose the one that comes first in lexicographical order. We therefore propose to classify and label the sequences with the lexicographically minimal rotation of the most prevalent k-mer. This way the DNA sequence gets labeled by the most prevalent sequence motif, regardless of its exact distribution. In R, the classification into labelled classes was achieved by the following $\mathrm{R}$ code:

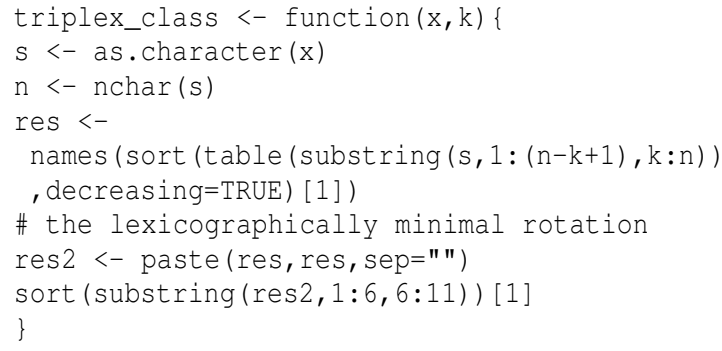

One can then easily annotate sequences identified by triplex.search(), and stored in the variable $t c$, simply by calling

sort (table (sapply (tc, triplex_class, 6)) 
, decreasing=TRUE)

This novel method of annotation will probably need to be further refined, since in its proposed form it only works well if the value of $k$ used in the analysis is equal to the intrinsic periodicity of the analysed sequence. Using hexamers succesfully captures periods of 1,2,3 and 6 but may give fragmented results for other periods. We presently solve this by manually choosing a class name based on an inspection of the hexamer, such as CTT/GAA (see Table 1).

\subsection{Insertions or deletions in potential triplex sequences}

The output of triplex.alignment() contains the designation of individual H-DNA strands. They are labelled as plus, minus, par+, par-, apar+, aparand loop. We used the script count_chars.R to determine the frequency of insertions/deletions (symbol "-") or other symbols in the aligned DNA strands. We expected different tolerance for insertions/deletions between strands, because the properties of the original duplex (strands plus and minus may be quite different from the properties of the third DNA strand attached via Hoogsteen or reverse Hoogsteen bonds. The counted insertions were compared to the total length of each type of strand (regular expression "." for any symbol).

\section{RESULTS}

A series of calculations were carried out as described in section 2 to understand the distribution of PTS in the human genome and determine the properties of these sequences.

\subsection{Distribution of potential triplex sequences in the human genome}

To obtain an overall picture of how potential triplex sequences (PTS) are distributed in the human genome, we first compared the PTS positions with the positions of general annotated genome features, such as protein-coding sequences, promoters, introns and intergenic regions. There is a clear preference of PTS to be present in promoters or intergenic regions with close-to-predicted content in introns and strong avoidance of exons, including 5' - and 3'-UTRs (Figure 1).

Because the intergenic regions in the human genome are also known to harbor a high number of repetitive sequences (e.g. 10\% Alu (SINE), 15\% L1 (LINE), 8\% LTR retrotransposons) we calculated the

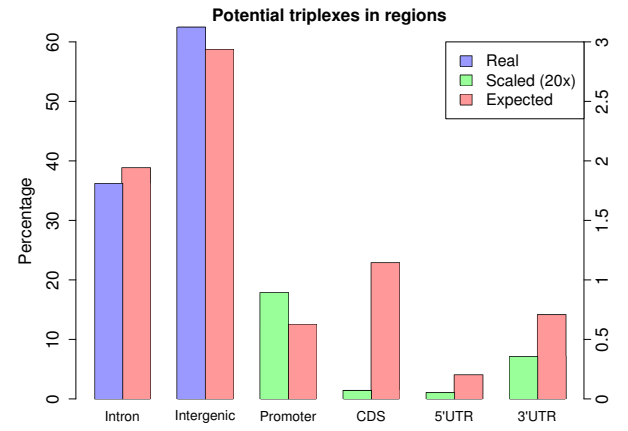

(a)

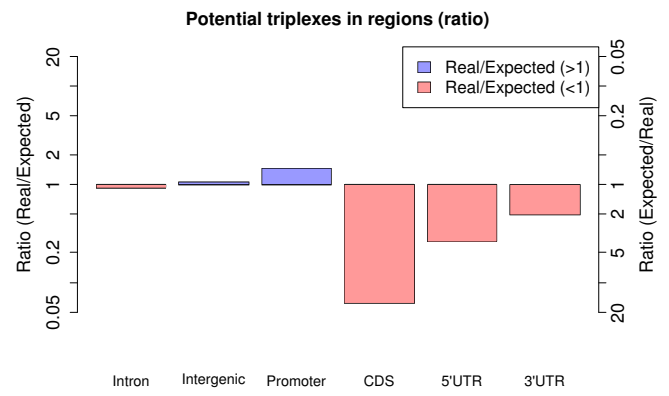

(b)

Figure 1: Distribution of potential triplex sequences (PTS) in genes and intergenic regions. The figure (a) shows the percentage of all elements in a given region. The four bars on the right side, namely Promotor, CDS, 5'UTR and 3'UTR, were zoomed in $20 \times$ and supplemented with its own axis. The figure (b) shows the ratio between real and expected counts. The expected number of triplexes was estimated from triplex density and the overall length of sequence in a given category.

number of PTS associated with individual repetitive sequence classes (Figure 2(a)) and families (Figure 2(b)). This analysis shows that only a limited number of repeat classes and families associate with $\mathrm{H}$ DNA more frequently than expected from genome averages.

To allow better comparison across families and classes that would not depend on repeat size and frequency of occurrence, we calculated the ratio between real and expected counts. In both types of analysis, there is a strong enrichment of PTS sequences in low complexity sequences and simple repeats (Figure 2(c)). Such findings are compatible with the limited number of nucleotide triplets found in stable H-DNA (Soyfer and Potaman, 1995) (Lexa et al., 2011) and the general requirement for polypurine and polypyrimidine tracts in triplexes. We also found SVA and to a limited extent also SINE and scRNA elements to have above average PTS association (Figure 2(c)). Of these, specifically SVA and Alu sequences showed above average association (Figure 2(d)).

We also looked whether there was a difference be- 


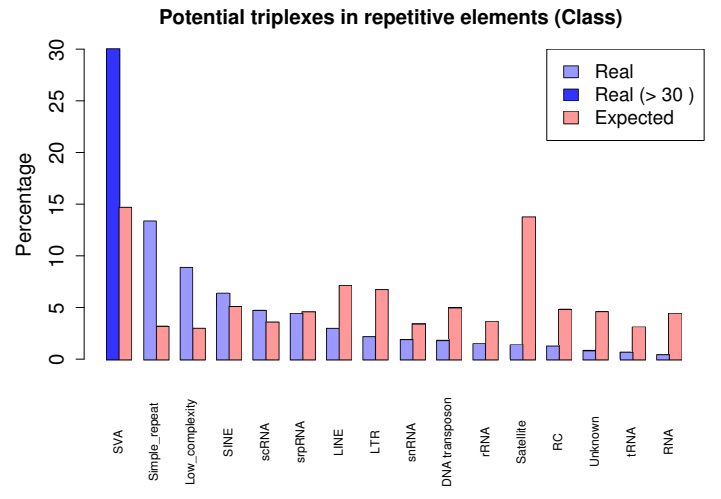

(a) Class

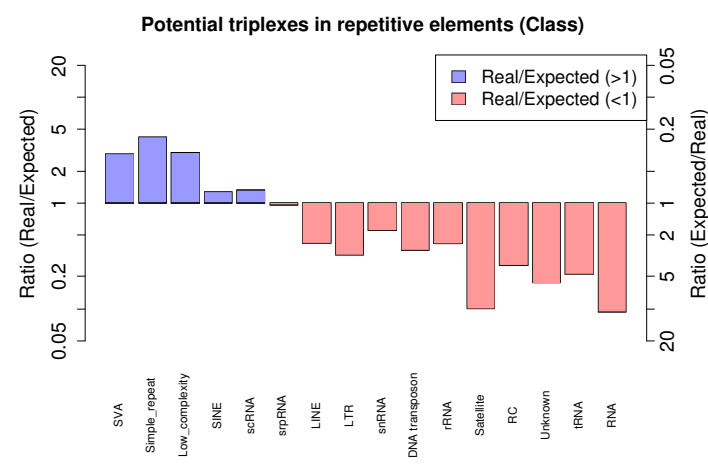

(c) Class ratio

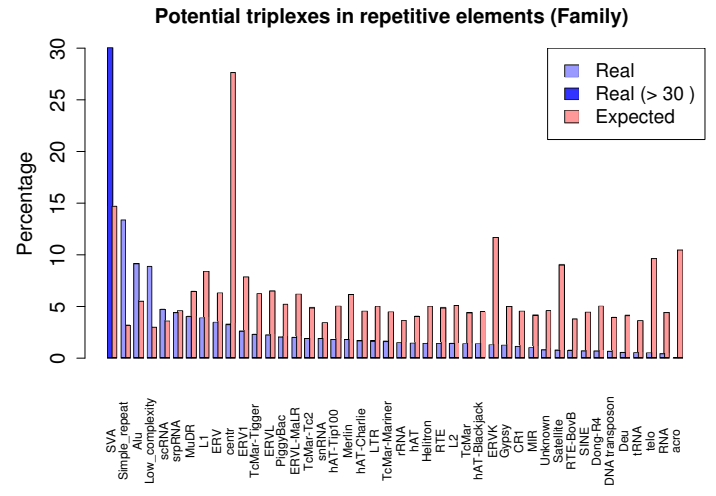

(b) Family

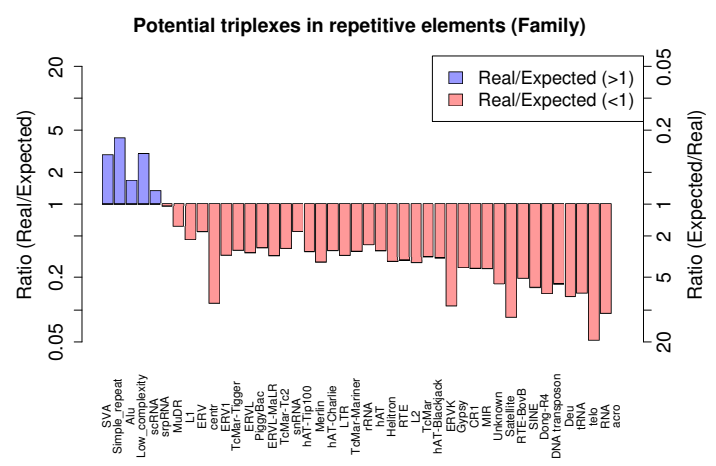

(d) Family ratio

Figure 2: Association of potential triplex sequences (PTS) with different families of repetitive sequences identified by RepeatMasker. The figures (a) and (b) show the percentage of all elements in a given family harboring at least one PTS. The figures (c) and (d) show ratio between real and expected counts test The expected number of triplexes was estimated from triplex density and the overall length of sequences of a given family.

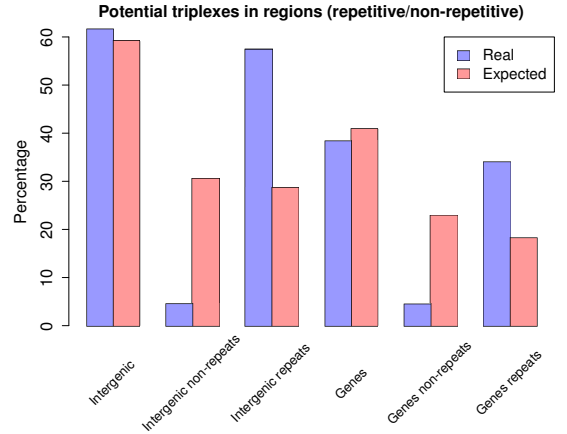

Figure 3: Occurrence of repeat and non-repeat-associated potential triplex sequences (PTS) in different regions of the human genome. Data shown as percentage of all PTS in the genome. The expected percentages of triplexes were estimated from average triplex density and the overall length of sequences of a given genomic region.

tween PTS sequences found in the different regions of the human genome. While the frequency of PTS in intergenic regions was slightly higher than in genes, were they prevailed in introns, they were equally associated with repeats in both parts, introns and inter- genic (Figure 3).

\subsection{Classification of potential triplexes by sequence composition}

To classify the detected PTS, we counted all nucleotide hexamers in their sequence and determined the lexicographically minimal rotation of the prevalent hexamer as described in section 2. This analysis revealed the presence of six main categories of PTS in the human genome (Table 1). In the order of prevalence, these were classes labelled by us as $T / A$ (45.8\%), CT /GA (20.6\%), CTT / GAA (14.6\%), $C C T / G G A$ (13.1\%), C/G (3.6\%), CA/GT (1.6\%) and $T A / T A(0.5 \%)$. The remaining PTS constituted only $0.3 \%$ of detected sequences. 
Table 1: Classification of PTS by sequence composition and the occurrence of different composition classes in the human genome.

\begin{tabular}{l|c|c|l}
\hline Hexamer & Count & {$[\%]$} & Class/Composition \\
\hline TTTTTT & 4360 & 16.6 & T/A \\
AAAAAA & 4346 & 16.5 & T/A \\
AAAAAG & 1993 & 7.6 & T/A \\
CTCTTT & 1564 & 5.9 & CT/GA \\
CTCTCT & 1470 & 5.6 & CT/GA \\
AGAGAG & 1444 & 5.5 & CT/GA \\
CTTTTT & 1337 & 5.1 & T/A \\
CCCCTT & 992 & 3.8 & CCT/GGA \\
AAAAGG & 954 & 3.6 & CTT/GAA \\
AAAGAG & 950 & 3.6 & CT/GA \\
CCCCCT & 624 & 2.4 & C/G \\
AGAGGG & 568 & 2.2 & CCT/GGA \\
AAGGGG & 528 & 2.0 & CCT/GGA \\
CCTCCT & 520 & 2.0 & CCT/GGA \\
CCCTCT & 508 & 1.9 & CCT/GGA \\
CCTTTT & 480 & 1.8 & CTT/GAA \\
CTTCTT & 439 & 1.7 & CTT/GAA \\
CCTTCT & 417 & 1.6 & CTT/GAA \\
AAGAAG & 410 & 1.6 & CTT/GAA \\
AAGAGG & 345 & 1.3 & CTT/GAA \\
AGGAGG & 326 & 1.2 & CCT/GGA \\
AAGGAG & 314 & 1.2 & CTT/GAA \\
AGGGGG & 311 & 1.2 & C/G \\
CCTCTT & 285 & 1.1 & CTT/GAA \\
GTGTGT & 225 & 0.9 & CA/GT \\
ACACAC & 187 & 0.7 & CA/GT \\
ATATAT & 144 & 0.5 & TA/TA \\
AAAGGG & 109 & 0.4 & CTT/GAA \\
CCCTTT & 85 & 0.3 & CTT/GAA \\
OTHER & 68 & 0.3 & - \\
\hline
\end{tabular}

\subsection{Tolerance of different potential triplex classes and aligned DNA strands for insertions}

We hypothesized that triplex sequences have asymmetric tolerance for insertions/deletions. Because the third strand binds to a DNA duplex in its major grove with lower stringency than seen in WatsonCrick basepaired duplex, the third strand may be able to accept insertions, but not deletions when aligned to the duplex with triplex.alignment(). Although the ability of H-DNA to accept mismatches, let alone indels, is highly questionable, we still carried out this calculation, counting the occurrence of the "-" symbol in different strands and counting the overall length (Table 2). We found that only $0.75 \%$ of positions in PTS were insertions/deletions in PTS scoring 25 or more. Moreover, when we compared the occurence of deletions in the different types of PTS third strand to the frequencies observed in the duplex at various score thresholds, we found the percentage to be lower in parallel strands (0.87-0.97\%) and much lower in antiparallel strands $(0.16-0.22 \%)$ (Table 3$)$. These data appear to support our hypothesis of asymmetrical insertion/deletion distribution among DNA strands in potential triplexes.

\section{DISCUSSION}

We have taken a closer look at the output of the $\mathrm{R} /$ Bioconductor triplex search package when ran against the human genome DNA sequence. In terms of search results, we were interested to see the different categories of human sequences that associate with potential intramolecular triplexes. The slight overrepresentation of PTS in non-coding sequences and clear absence from coding sequences seen in Figure 1 led us to focus on intergenic DNA, promoters and introns in more detail (Figure 2(a), 2(b)). H-DNA has been found in promoters of genes involved in disease (Bissler, 2007) and cell signalling and communication (Bacolla et al., 2006).

There is a common theme to the majority of PTS occurrences we observed in human DNA. Inspection of Figure 2(b)-2(d) reveals the presence of PTS in or near Alu, scRNA and simple repeat or low complexity sequences. Alu sequences are short non-autonomous retrotransposons (SINE) driven by the L1 LINE element protein machinery (Dewannieux et al., 2003) thought to have emerged in primate as duplication descendants of 7SL sc RNA (Kriegs et al., 2007). SVA repeats, which contained more then twice the number of PTS than expected by chance are also strongly associated with PTS. Perhaps not surprisingly, even SVA elements are evolutionarily related to SINE and Alu sequences. Their sequence is chimeric and contains two sequences of SINE origin separated by a variable number tandem repeat (Savage et al., 2013). According to our study, a large proportion of PTS in the human genome can therefore be directly attributed to the proliferation of SINE elements, especially Alu.

Upon first inspection, it becomes clear that most of the above-mentioned associations are caused by the presence of the polyA tail in SINE elements. Because the poly-A tail is mainly described as a feature circumventing the problematic polyadenylation in RNA polymerase III transcripts (Roy-Engel, 2012), there is a possibility that these sequences do not form any functionally or evolutionarily meaningful DNA structures, such as H-DNA. On closer inspection, however, we notice that the same classes of repeats are also en- 
riched for other PTS sequences, raising the possibility that triplex formation plays a biological role in the repeat life cycles also at the DNA level. This could also mean a dual role for the Alu poly-A tail. For example, (Dewannieux and Heidmann, 2005) mention a 15-50 nucleotide range for increasing effect of the poly-A tail, a range that also coincides with cited oligonucleotide lengths for successful H-DNA formation (Buske et al., 2011). (CT) ${ }_{n}$ tandem repeats have also been implicated in tandem array maintenance (Bailey et al., 2013), the mechanism and its dependence on triplex formation is, however, presently unknown. (Brereton et al., 1993) showed that the Arich sequence in a human Alu element can form an intramolecular triplex in vitro.

Given the presence of PTS in Alu and SVA repeats in human, that have evolved as dimers (the former) and dimer of dimers (the latter) of ancient RNA, there is a possibility for intramolecular triplexes to aid the recombination processes leading to chimeric sequences. There are indeed many reports of H-DNA occurrence near recombination hotspots (Napierala et al., 2004).

Because of the high Alu content of the human genome, the presence of PTS in Alu elements biased our estimates of expected PTS association frequency with other elements. Upon subtracting these from our results, several other classes get into the "above-expected" occurrence territory, namely the L1 retrotransposon and MuDR DNA transposon as well as snRNA which often contains a (CT)n dinucleotide tandem repeat.

We have also noticed a high occurrence of PTS in the miRNA class of RNAs (data not shown). Kanak and colleagues (Kanak et al., 2010) recently reported the discovery of a set of miRNA sequences that could form triplexes at HIV target sites and suppress its retroviral activity. An increased presence of PTS sequences has recently been reported in the 5' and 3'UTR of plant retroelements, probably analogous to the reported 3'-UTR HIV regulatory region.

Probably the second most typical location for PTS in our study were the promoters of genes. The formation of special DNA structures at sequences such as PTS studied in this paper may create structurally distinct features providing possibilities for specific DNA-binding proteins to recognize locations in the genome for gene regulation or chromatin organization. For example, triplex DNA has been found to be incompatible with nucleosome formation and may act as a nucleosome barrier (Westin et al., 1995). They are often found near recombination and mutation hotspots (Napierala et al., 2004)(Akman et al., 1991). This may be related to the inevitability of single-stranded DNA stretches at or near the triplexes. Association of PTS with certain types of repeat elements could not only suggest a possible function in the repeat "life cycle" but also a possible positive selection for repeats with such association, if the presence of triplexes was required at several locations of the host genome.

Among the typical hexamers found in triplexes, we identified a minor group wih prevailing CA/GT dinucleotide repeats. Although this combination does not meet the often cited requirement for homopurine and homopyrimidine tracts in H-DNA, it may actually form intramolecular triplexes in combination with other base triplets, as observed by (Gowers and Fox, 1998). Our extremely low counts (Table 1) seem to support the notion that if G.T:A and T.A:T triplets occur in triplexes, they are most likely to be mixed with other nucleotide combinations.

\section{CONCLUSIONS}

In this paper we examined the types of sequences that can be identified in the human genome DNA sequence with triplex DNA detection software, namely the R/Bioconductor package triplex-1.0.10 and its triplex.search() function. The presented results examine the usability of the software for genome studies as well as some basic properties of the identified potential triplex sequences (PTS). We found that most of the triplex-forming potential of the human genome is concentrated in simple repeats and flanking regions of repetitive and other genome elements descending from 7SL RNA, especially Alu and SVA repeats. We also found potential triplex-forming sequences in the miRNA class of RNA genes. Alu elements are known to contain or flank adenine homonucleotide tracts which replace polyadenylation of its RNA, but could also carry out a DNA-based function involving $\mathrm{H}$-DNA formation.

We propose a computational rule to automatically classify triplex-forming sequences according to the most prevalent $k$-mer present in their sequence. For unambiguity, we include the search for a lexicographically minimal rotation before assigning the name. After applying this principle we see that the majority of human PTS fall into four main classes based on their nucleotide composition (T/A - 45.8\%; CT/GA 20.6\%; CTT/GAA - $14.6 \%$ and CCT/GGA - 13.1\%). We also characterized the detected PTS based on deletions found in alignments of the third triplex strand to the DNA duplex, sowing that deletions are present less frequently in the third strand, especially in antiparallel PTS. 
In terms of biological relevance, our studies of PTS suggest they are positioned non-randomly in the genome, their sequences fall into a small number of distinct classes and some of them are associated with specific types of repeats. Their strand bias for insertions or deletions suggests that these sequences may indeed form the predicted structures. In future it would be desireable to single out specific combination of repeat types and PTS classes, prove the existence of triplex formation in each case and systematically search for proteins that could interact with such structures and provide a more precise clue to their specific biological function.

\section{ACKNOWLEDGEMENTS}

This work was supported by research grants from the European Social Fund (CZ.1.07/2.3.00/20.0189) to M.L., 13-36108S (Grant Agency of Science of CR) to M.B., by IT4Innovations project, reg. no. CZ.1.05/1.1.00/02.0070 funded by the EU Operational Programme, by MSMT Grant No.0021630528 and by BUT grant FIT-S-11-1.

\section{REFERENCES}

Aboyoun, P., Pages, H., and Lawrence, M. (2013). Genomicranges: Representation and manipulation of genomic intervals. Technical Report $\mathrm{R}$ package version 1.10.7.

Akman, S. A., Lingeman, R. G., Doroshow, J. H., and Smith, S. S. (1991). Quadruplex dna formation in a region of the trna gene supf associated with hydrogen peroxide mediated mutations. Biochemistry, 30(35):8648-8653.

Arora, A., Dutkiewicz, M., and Scaria, V. (2008). Inhibition of translation in living eukaryotic cells by an rna gquadruplex motif. RNA, 14:1290-1296.

Bacolla, A. and Wells, R. (2004). Non-b dna conformations, genomic rearrangements, and human disease. Journal of Biological Chemistry, 279:47411-47414.

Bacolla, A., Wojciechowska, M., Kosmider, B., Larson, J. E., and Wells, R. D. (2006). The involvement of non-b dna structures in gross chromosomal rearrangements. DNA Repair, 5:1161-1170.

Bailey, A. D., Pavelitz, T., and Weiner, A. M. (2013). The microsatellite sequence (ct)n.(ga)n promotes stable chromosomal integration of large tandem arrays of functional human u2 small nuclear rna genes. Molecular and Cellular Biology, 18(4):2262-2271.

Bissler, J. J. (2007). Triplex dna and human disease. Frontiers in Bioscience, 12:4536-4546.

Brereton, H., Firgaira, F., and Turner, D. (1993). Origins of polymorphism at a polypurine hypervariable locus. Nucleic Acids Research, 21(11):2563-2569.
Buske, F. A., Bauer, D. C., Mattick, J. S., and Bailey, T. L. (2012). Triplexator: detecting nucleic acid triple helices in genomic and transcriptomic. Genome Research, 22(7):1372-1381.

Buske, F. A., Mattick, J. S., and Bailey, T. L. (2011). Potential in vivo roles of nucleic acid triple-helices. $R N A$ Biology, 8(3):427-439.

Cer, R. Z., Bruce, K. H., Mudunuri, U. S., Yi, M., Volfovsky, N., Luke, B. T., Bacolla, A., Collins, J. R., and Stephens, R. M. (2011). Non-b db: a database of predicted non-b dna-forming motifs in mammalian genomes. Nucleic Acids Research, 39(Database issue):D383-D391.

Dewannieux, M., Esnault, C., and Heidmann, T. (2003). Line-mediated retrotransposition of marked alu sequences. Nature Genetics, 35:41-48.

Dewannieux, M. and Heidmann, T. (2005). Role of poly(a) tail length in alu retrotransposition. Genomics, 86(3):378-381.

Dixon, B., Lu, L., Chu, A., and Bissler, J. (2008). Recq and recg helicases have distinct roles in maintaining the stability of polypurine.polypyrimidine sequences. Mutation Research, 643:20-28.

Durinck, S., Spellman, P., Birney, E., and Huber, W. (2009). Mapping identifiers for the integration of genomic datasets with the $\mathrm{r} /$ bioconductor package biomart. $\mathrm{Na}$ ture Protocols, 4:1184-1191.

Gowers, D. and Fox, K. (1998). Triple helix formation at (at)n adjacent to an oligopurine tract. Nucleic Acids Research, 26(16):3626-3633.

Hon, J., Martinek, T., Rajdl, K., and Lexa, M. (2013). Triplex: an r/bioconductor package for identification and visualization of potential intramolecular triplex patterns in dna sequences. Bioinformatics, 29(15):1900-1901.

Kanak, M., Alseiari, M., Balasubramanian, P., Addanki, K., Aggarwal, M., Noorali, S., Kalsum, A., Mahalingam, K., Pace, G., Panasik, N., and Bagasra, O. (2010). Triplex-forming micrornas form stable complexes with hiv-1 provirus and inhibit its replication. Applied Immunohistochemistry and Molecular Morphology, 18(6):532-545.

Karolchik, D., Hinrichs, A. S., Furey, T. S., Roskin, K. M., Sugnet, C. W., Haussler, D., and Kent, W. J. (2004). Ucsc table browser data retrieval tool. Nucleic Acids Research, 32(Database issue):D493-D496.

Kriegs, J. O., Churakov, G., Jurka, J., Brosius, J., and Schmitz, J. (2007). Evolutionary history of 7sl rnaderived sines in supraprimates. Trends in Genetics, 23(4):158-161.

Lawrence, M., Huber, W., Pags, H., Aboyoun, P., Carlson, M., Gentleman, R., Morgan, M. T., and Carey, V. J. (2013). Software for computing and annotating genomic ranges. PLoS Computational Biology, 9(8):e1003118.

Lexa, M., Kejnovsky, E., Steflova, P., Konvalinova, H., Vorlickova, M., and Vyskot, B. (2013). Quadruplexforming sequences occupy discrete regions inside plant ltr retrotransposons. Nucleic Acids Research, page 10.1093/nar/gkt893 (ePub). 
Lexa, M., Martinek, T., Burgetova, I., Kopecek, D., and Brazdova, M. (2011). A dynamic programming algorithm for identification of triplex-forming sequences. Bioinformatics, 27(18):2510-2517.

Maizels, N. and Gray, L. (2013). The g4 genome. PLoS Genetics, 9(4):e1003468.

Napierala, M., Dere, R., Vetcher, A. A., and Wells, R. D. (2004). Dna replication repair and recombination: Structure-dependent recombination hotspot activity of gaattc sequences from intron 1 of the friedreich's ataxia gene. The Journal of Biological Chemistry, 279:6444-6454.

Pages, H. (2013). Bsgenome: Infrastructure for biostringsbased genome data packages. Technical Report $\mathrm{R}$ package version 1.26.1.

Pages, H., Aboyoun, P., Gentleman, R., and DebRoy, S. (2013). Biostrings: String objects representing biological sequences, and matching algorithms. Technical Report R package version 2.26.3.

Rich, A. and Zhang, S. (2008). Timeline: Z-dna: the long road to biological function. Nature Reviews Genetics, 4:566-572.

Roy-Engel, A. M. (2012). A tale of an a-tail. the lifeline of a sine. Mobile Genetic Elements, 2(6):282-286.

Sarkies, P., Murat, P., Phillips, L., Patel, K., Balasubramanian, S., and Sale, J. (2012). Fancj coordinates two pathways that maintain epigenetic stability at gquadruplex dna. Nucleic Acids Research, 40(4):14851498.

Savage, A. L., Bubb, V. J., Breen, G., and Quinn, J. P. (2013). Characterisation of the potential function of sva retrotransposons to modulate gene expression patterns. BMC Evolutionary Biology, 13(101).

Schwab, R. A., Nieminuszczy, J., Shin-ya, K., and Niedzwiedz, W. (2013). Fancj lets chromatin stay true. Journal of Cell Biology, 201:33-48.

Soyfer, V. and Potaman, V. (1995). Triple-helical nucleic acids. Springer-Verlag, Heidelberg.

Westin, L., Blomquist, P., and Milligan, J. F. e. a. (1995). Triple helix dna alters nucleosomal histone-dna interactions and acts as a nucleosome barrier. Nucleic Acids Reserch, 23:2184-2191.

Zhao, J., Bacolla, A., Wang, G., and Vasquez, K. (2010). Non-b dna structure-induced genetic instability and evolution. Cellular and Molecular Life Sciences, 67(1):43-62. 
Table 2: The number of deletions counted in different strands of PTS in the human genome DNA sequence and the total number of PTS strands examined.

\begin{tabular}{r|rrrrr|rrrrr}
\hline & \multicolumn{4}{|c}{ Number of deletions } & \multicolumn{4}{c}{ Number of PTS strands of a given type } \\
\hline Score & duplex & para+ & para- & anti+ & anti- & duplex & para+ & para- & anti+ & anti- \\
\hline$>25$ & 7953 & 3053 & 2931 & 202 & 170 & 956452 & 378942 & 357804 & 109834 & 109872 \\
$>35$ & 7443 & 2934 & 2834 & 116 & 105 & 853345 & 365429 & 345811 & 70800 & 71305 \\
$>50$ & 3972 & 1630 & 1624 & 8 & 10 & 425395 & 206228 & 200898 & 8985 & 9284 \\
$>70$ & 1001 & 442 & 437 & 0 & 0 & 148244 & 72251 & 73930 & 897 & 1166 \\
\hline
\end{tabular}

Table 3: The frequency and relative occurrence of deletions in DNA strands of different classes in human PTS.

\begin{tabular}{c|ccccc|cc}
\hline & \multicolumn{4}{|c}{$100 *$ deletions/strand } & \multicolumn{2}{c}{ relative to duplex } \\
\hline Score & duplex [\%] & para+ [\%] & para- [\%] & anti+ [\%] & anti- [\%] & para/duplex & anti/duplex \\
\hline$>25$ & 0.83 & 0.81 & 0.82 & 0.18 & 0.15 & 0.98 & 0.19 \\
$>35$ & 0.87 & 0.80 & 0.82 & 0.16 & 0.15 & 0.93 & 0.17 \\
$>50$ & 0.93 & 0.79 & 0.81 & 0.09 & 0.11 & 0.86 & 0.10 \\
$>70$ & 0.68 & 0.61 & 0.59 & 0.00 & 0.00 & 0.88 & 0.00 \\
\hline
\end{tabular}

